\title{
RELAÇÃO ENTRE A QUALIDADE DO PRÉ-NATAL E DESFECHO MATERNO FETAL EM UMA MATERNIDADE PÚBLICA EM MACAPÁ
}

\section{ARTIGO ORIGINAL}

CORDEIRO, Ângela Cristina da Silva ${ }^{1}$

RÊGO, Aljerry²

OLIVEIRA, Vitor Fernando dos Santos ${ }^{3}$

GOMES, Rayllane Barbosa ${ }^{4}$

CORDEIRO, Ângela Cristina da Silva. Et al. Relação entre a qualidade do pré-natal e desfecho materno fetal em uma maternidade pública em Macapá. Revista Científica Multidisciplinar Núcleo do Conhecimento. Ano 04, Ed. 07, Vol. 11, pp. 5366. Julho de 2019. ISSN: 2448-0959

\section{RESUMO}

A assistência pré-natal é um importante componente da atenção à saúde das mulheres no período gravídico-puerperal. O objetivo desse estudo foi analisar a adequação do pré-natal e fatores de risco associados à prematuridade em uma capital da região Norte do Brasil. Método: a pesquisa se caracteriza como transversal qualitativo. Os dados foram obtidos através de entrevista e análise da caderneta da gestante de 908 puérperas, atendidas no Hospital da Mulher e Maternidade Mãe luzia no período de abril a julho de 2019. Neste estudo foi utilizado o nível de confiança

${ }^{1}$ Médica. Residente de Ginecologia e Obstetrícia pela Universidade Federal do Amapá.

2 Médico. Mestre em Ginecologia pela USP. Professor do curso de Medicina na Universidade Federal do Amapá.

${ }^{3}$ Acadêmico de Medicina pela Universidade Federal do Amapá.

${ }^{4}$ Acadêmica de Medicina pela Universidade Federal do Amapá. 
usual de $5 \%(0,05)$, deste modo para análise dos testes estatísticos consideramos $p$ valor $<0,05$, como significativo, deste modo desconsideraremos a hipótese de igualdade (Ho). Resultados: faixa etária predominante foi $\geq 20$ anos $(79,6 \%)$; escolaridade ensino fundamental (38,7\%); situação conjugal casada (83,7\%); renda familiar < 1 salário mínimo (50\%); idade gestacional $\geq 37$ semanas $(84,1 \%)$; registro de resultado de ultrassonografia $>14$ semanas ( $60,7 \%)$; realizou os exames preconizados pelo Ministério da Saúde ( $81,8 \%)$, repetiu os exames no terceiro trimestre (33\%); realizaram 3 ou mais consultas médicas (32,9\%); realizaram 3 ou mais consultas com enfermeiro $(51,5 \%)$. Concluímos que o desfecho neonatal desfavorável (Apgar Ruim) em parturientes atendidas em uma maternidade pública do Amapá foi estatisticamente significativo nas seguintes situações: a prematuridade, parto cesariano, necessidade de UTI neonatal e baixo peso ao nascer.

Palavras-chaves: assistência pré-natal, prematuridade, saúde materno-infantil.

\section{INTRODUÇÃO}

A assistência ao pré-natal é fundamental para que se obtenha um resultado positivo no desfecho de gestação, e a sua qualidade está relacionada com a disponibilidade de recursos em âmbito gerencial e assistencial, bem como ao desenvolvimento de ações de forma rotineira, obedecendo padrões técnico-científicos de qualidade ${ }^{6}$.

O Programa de Humanização no Pré-natal e Nascimento, do Ministério da Saúde, preconiza que o adequado acompanhamento pré-natal é feito com, no mínimo, seis consultas de acompanhamento, preferencialmente, uma no primeiro trimestre, duas no segundo trimestre e três no terceiro trimestre da gestação; e que a primeira consulta seja realizada até o $4^{\circ}$ mês de gestação ${ }^{3}$.

No Brasil, ainda que haja, uma cobertura significativa da assistência pré-natal, superior a $90 \%$, esta ocorre de maneira inadequada, em que apenas $60 \%$ recebem orientações sobre a realização de exames, parto e aleitamento. Problemas pessoais, dificuldades de acesso ou de diagnóstico de gravidez constituem uma barreira para não realização e para o início tardio do pré-natal ${ }^{10}$. 
A prematuridade constitui-se em um grande problema de Saúde Pública, por se tratar de um determinante de morbimortalidade neonatal, e a assistência ao pré-natal é elencada entre estes fatores de risco para sua ocorrência. Quando prestada com qualidade à gestante, isto é, quando há infraestrutura adequada (recursos físicos, materiais, humanos e financeiros) com as devidas orientações e condutas poderá se obter melhores resultados na assistência ao parto e ao nascimento ${ }^{4}$.

O objetivo deste trabalho foi analisar a adequação do pré-natal e fatores de risco associados à prematuridade em uma capital da região Norte do Brasil, avaliar o perfil epidemiológico das gestantes internadas em trabalho de parto, a qualidade do prénatal através da análise da caderneta da gestante e a relação entre a prematuridade e qualidade do pré-natal.

\section{METODOLOGIA}

Trata-se de um estudo do tipo transversal qualitativo, realizado através de entrevistas e análise da caderneta da gestante em puérperas internadas no Hospital da Mulher Mãe Luzia localizado na cidade de Macapá, Amapá, no período de abril a julho de 2019, após consentimento informado e autorização das puérperas. Na seleção da amostra, foram obedecidos os seguintes critérios de inclusão: partos com idade gestacional acima de 22 semanas. Foram excluídas as puérperas que tiveram partos gemelares, as que não tinham caderneta da gestante, as puérperas com menos de 22 semanas de gestação ou procedentes de outros estados. A amostra final analisada é formada por 908 pacientes.

Para a identificação da idade gestacional de nascimento adotou-se aquela registrada pelo obstetra no prontuário da puérpera, foram definidos como puérperas de recémnascidos prematuros aquelas com idade gestacional inferior a 37 semanas. Enquanto que o peso, o escore de APGAR e a necessidade de UTI dos recém-nascidos, utilizouse $o$ registro feito pela pediatria. 
A coleta de dados foi realizada por meio de entrevista estruturada com as puérperas em um prazo de até 48 horas após o parto. Os dados foram coletados pelos pesquisadores responsáveis pelo estudo.

As variáveis abordadas na entrevista envolveram características socioeconômicas e demográficas: região da residência (zona norte, zona sul da capital, interior do estado), idade (10-14 anos; 15-19 anos; 20-24 anos; 25-24 anos; 35 ou mais anos), raça (branca, preta, parda, amarela ou indígena), escolaridade (ensino fundamental incompleto, ensino fundamental completo, ensino médio incompleto, ensino médio completo, ensino superior incompleto, ensino superior completo), renda familiar $(<1$ salário mínimo, de 1 a 2 salários mínimos ou > 3 salários mínimos), situação conjugal ( com companheiro ou sem companheiro). História reprodutiva e da gestação atual: número de gestações anteriores ( 1,2 ou mais que 3 ), desfechos negativos de gestações anteriores, tipo de parto em gestação anterior, idade gestacional no momento do parto, registro de resultados de ultrassonografia na caderneta da gestante (sem registro, registro com < 14 semanas de gestação ou registro com idade gestacional > 14 semanas), realizou os exames preconizados pelo Ministério da Saúde (hemograma, tipagem sanguínea, sorologia para toxoplasmose, hepatite B e $\mathrm{C}$, sífilis, HIV, glicemia de jejum), e se os exames foram repetidos no terceiro trimestre. Foram avaliados o número de consultas realizados por médicos (nenhuma, 1, 2, 3 ou mais), número de consultas realizados por enfermeiros (nenhuma, 1, 2 , 3 ou mais), o tipo de parto ( vaginal ou cesárea) e o que motivou o parto cesáreo e o desfecho materno ( necessidade de UTI ou óbito).Também feito análise do escore de APGAR (no primeiro e quinto minuto após o nascimento), peso do recém-nascido (RN), necessidade de UTI neonatal ou óbito neonatal.

Após a coleta as informações foram inseridas em um banco de dados do software Statistical Package for the Social Sciences (SPSS) versão 22. As tabelas e os gráficos foram construídos com o auxílio do software Microsoftware Excel 2016. Foi utilizado o nível de confiança usual de $5 \%(0,05)$, deste modo para análise dos testes estatísticos consideramos $p$-valor $<0,05$, como significativo. 
Visando cumprir todos os preceitos éticos e legais das pesquisas que envolvem seres humanos, o projeto foi aprovado pelo Comitê de Ética e Pesquisa (CEP) da Universidade Federal do Amapá (CAAE 13259519.6.0000.0003). Todas as participantes desta pesquisa assinaram o Termo de Consentimento Livre e Esclarecido (TCLE), além disso, foi requerido permissão de acesso aos dados das pacientes ao gestor da maternidade onde se realizou o estudo.

\section{RESULTADOS E DISCUSSÃO}

No período de abril a julho de 2019, foram coletados dados de 908 puérperas no Hospital da Mulher Mãe Luzia, destas $764(84,1 \%)$ com idade gestacional maior ou igual a 37 semanas de gestação e $144(15,9 \%)$ pacientes com idade gestacional inferior a 37 semanas de gestação (Tabela 1). Enquanto um estudo realizado no estado de Santa Catarina em 2005 mostrou uma prevalência de prematuridade igual a $6,1 \%^{2}$. Estudos em diversos países encontraram, entre os fatores de risco para o nascimento prematuro a inadequação do cuidado pré-natal ${ }^{2}$.

O perfil materno foi dividido quanto à localização da moradia: 39,4\% residem na zona sul da capital, $35,6 \%$ residem na zona norte da capital e $25 \%$ no interior do estado.

Com relação à idade, $79,6 \%$ adultas e $20,4 \%$ pacientes jovens, observamos, que quanto à raça $80,6 \%$ são de raça parda, 13,8\% raça preta, 4,2\% raça branca, $1,0 \%$ raça amarela e $0,4 \%$ eram indígenas. Em relação a escolaridade, os resultado indicaram que: $32 \%$ estudaram até o ensino fundamental incompleto, 31,8\% concluíram o ensino médio, $18,2 \%$ ensino médio incompleto, 7,0\% completaram o ensino superior, $6,7 \%$ estudaram até o ensino fundamental completo e $4,2 \%$ ensino superior incompleto.

No que se refere a renda familiar $50 \%$ da entrevistadas viviam com menos de 1 salário mínimos, 44,6\% declararam que a renda familiar variava de 1 a 2 salários mínimos e apenas $5,4 \%$ com renda maior ou igual a 3 salários mínimos. Com relação a situação conjugal, encontramos: $83,7 \%$ com companheiros fixos e $16,3 \%$ sem companheiros fixos. 
Um estudo realizado no município de São Luís-MA evidenciou que o uso adequado e intensivo do pré-natal está intimamente relacionado com características socioeconômicas, como a alta escolaridade materna e a renda familiar ${ }^{3}$. Em estudo correlacionando ao estado civil da mãe, a mortalidade infantil e aspectos socioeconômicos demonstram que a taxa de mortalidade infantil é maior para as crianças de mães solteiras ${ }^{2}$.

Barreiras diversas para a não realização do pré-natal ou para o início precoce do acompanhamento foram identificadas, evidenciando as desigualdade sociais que persistem no país, com menor escolaridade, com maior número de gestações, e que residem nas regiões Norte e Nordeste ${ }^{10}$.

Levando em consideração a história reprodutiva das pacientes, 29,2\% primigestas, 28,2\% eram multíparas com 3 ou mais gestações, 24\% já tinham uma gestação anterior e 18,5\% com 2 gestações prévias.48,2\% declaram um desfecho negativo um gestação anterior e o tipo de parto que mais prevaleceu na história obstétrica das pacientes foi o parto normal: $48,1 \%$.

Com relação ao acompanhamento no pré-natal: $60,7 \%$ realizaram exame ultrassonográfico com idade gestacional superior a 14 semanas de gestação, 27,1\% com menos de 14 semanas e 12,2\% não realizaram o exame do decorrer da gestação. $81,8 \%$ realizaram os exames preconizados pelo Ministério da Saúde no primeiro trimestre e apenas $33 \%$ repetiram os exames no terceiro trimestre. Levando em consideração o número de consultas médicas $32,9 \%$ realizaram 3 ou mais consultas, 23,7\% não realizaram nenhuma consulta, 22,5\% realizaram 1 consulta e 20,9\% realizaram 2 consultas. Em contrapartida 51,5\% realizaram 3 ou mais consultas com o enfermeiro, 24,3\% realizaram 2 consultas, 20,8\% 1 consulta e apenas 3,3\% não realizaram consulta com o enfermeiro. Dados semelhantes demonstrado por Villas et al. ${ }^{10}$, seu estudo mostrou que $80 \%$ das puérperas realizou exame do primeiro trimestre, $40 \%$ repetiu exames no terceiro trimestre, $12,3 \%$ realizaram ultrassonografia obstétrica antes de 14 semanas, e 75,6\% das gestantes foram atendidas por um profissional médico. 
O principal objetivo dos exames de rotina no pré-natal é a identificação de intercorrências na gravidez com as intervenções oportunas para preservar a saúde da mãe e do feto, sendo a caderneta da gestante um dos principais instrumentos de comunicação entre as equipes de assistência ao pré-natal e ao parto, quanto aos problemas identificados e à condutas adotadas ${ }^{10}$.

Quanto ao desfecho materno da gestação $57 \%$ tiveram parto vaginal e $43 \%$ parto cesáreo. Não ocorrendo nenhum óbito e apenas $0,4 \%$ necessitaram de UTI.

Tabela 01- Características epidemiológicas e obstétricas de puérperas atendidas em uma maternidade da região Norte do Brasil. (PI)-2019. N:908

\begin{tabular}{|c|c|c|}
\hline & $\mathbf{N}$ & $\%$ \\
\hline \multicolumn{3}{|l|}{ Região da residência: } \\
\hline Zona Norte & 323 & $35,60 \%$ \\
\hline Interior do Estado & 227 & $25,00 \%$ \\
\hline Zona Sul & 358 & $39,40 \%$ \\
\hline \multicolumn{3}{|l|}{ Idade (anos) } \\
\hline$\leq 19$ anos (Jovem) & 185 & $20,4 \%$ \\
\hline$\geq 20$ anos (Adulto) & 723 & $79,6 \%$ \\
\hline \multicolumn{3}{|l|}{ Raça } \\
\hline Branca & 38 & $4,20 \%$ \\
\hline Preta & 125 & $13,80 \%$ \\
\hline Parda & 732 & $80,60 \%$ \\
\hline Amarela & 9 & $1,00 \%$ \\
\hline Indígena & 4 & $0,40 \%$ \\
\hline \multicolumn{3}{|l|}{ Escolaridade Materna } \\
\hline E. Fundamental (Incompleto) & 291 & $32,00 \%$ \\
\hline E. Fundamental (Completo) & 61 & $6,70 \%$ \\
\hline E. Médio (Incompleto) & 165 & $18,20 \%$ \\
\hline E. Médio (Completo) & 289 & $31,80 \%$ \\
\hline E. Superior (Incompleto) & 38 & $4,20 \%$ \\
\hline
\end{tabular}


E. Superior (Completo)

\section{Renda familiar}

$<1 \mathrm{SM}$

$1|-2| S M$

$\geq 3 S M$

Situação conjugal

Sem Companheiro

Com Companheiro

№ de gestações anteriores:

Nenhuma

$1^{\circ}$ Gestação

$2^{\circ}$ Gestação

$\geq 3^{\circ}$ Gestações

Desfechos negativos anteriores

Sim

Não

Nãos se aplica

Tipo de parto em gestação anterior

Cesáreo

Normal

Não se aplica
$64 \quad 7,00 \%$

$454 \quad 50,00 \%$

$40544,60 \%$

$49 \quad 5,40 \%$

$760 \quad 83,70 \%$

$265 \quad 29,20 \%$

$218 \quad 24,00 \%$

$168 \quad 18,50 \%$

$256 \quad 28,20 \%$

$205 \quad 22,60 \%$

$438 \quad 48,20 \%$

$265 \quad 29,20 \%$

\section{Idade gestacional no momento do parto}

$<37$ Semanas

$144 \quad 15,90 \%$

$\geq 37$ Semanas

$764 \quad 84,10 \%$

Registro de resultado de ultrassonografia ( no cartão)

Sem Registro

$111 \quad 12,20 \%$

Com registro $<14$ semanas

$246 \quad 27,10 \%$

Com registro $>14$ semanas

$55160,70 \%$

Fez todos os exames preconizados pelo MS?

\begin{tabular}{l|l|l} 
Sim & 743 & $81,80 \%$ \\
\hline Não & 165 & $18,20 \%$ \\
\hline
\end{tabular}




\begin{tabular}{|l|l|l|}
\hline \multicolumn{3}{|l|}{ Repetiu os exames no terceiro trimestre? } \\
\hline Sim & 300 & $33,00 \%$ \\
\hline Não & 608 & $67,00 \%$ \\
\hline Número de consultas médicas & & \\
\hline Nenhuma & 215 & $23,70 \%$ \\
\hline 1 Consulta & 204 & $22,50 \%$ \\
\hline 2 Consultas & 190 & $20,90 \%$ \\
\hline$\geq 3^{\circ}$ Consultas & 299 & $32,90 \%$ \\
\hline Número de consultas com enfermeiro & & \\
\hline Nenhuma & 30 & $3,30 \%$ \\
\hline 1 Consulta & 189 & $20,80 \%$ \\
\hline 2 Consultas & 221 & $24,30 \%$ \\
\hline$\geq 3^{\circ}$ Consultas & 468 & $51,50 \%$ \\
\hline Tipo de parto & & \\
\hline Vaginal & 518 & $57,00 \%$ \\
\hline Cesária & 390 & $43,00 \%$ \\
\hline Óbito materno: & & \\
\hline Sim & 0 & - \\
\hline Não & 908 & $100 \%$ \\
\hline Necessidade UTI adulto & 4 & $0,40 \%$ \\
\hline Sim & 905 & $99,60 \%$ \\
\hline Não & & \\
\hline
\end{tabular}

Fonte: Autor

Quanto ao desfecho neonatal (Tabela 2), no que se refere ao Apgar, levando em consideração a classificação da Sociedade Brasileira de Pediatria (SBP) que classifica o Apgar entre 8 a 10 ótimo a excelente, 5 a 7 regular e menos que 5 aqueles recémnascidos que precisa de auxílio médico especializado, no presente estudo, 92,1\% foram classificados com o Apgar ótimo/excelente no primeiro minuto e 97,5\% Apgar ótimo/excelente no quinto minuto. Com relação ao peso do nascimento $92,6 \%$ teve 
peso maior que $2.500 \mathrm{~g}$. Os resultados obtidos demonstraram também que $4,7 \%$ dos nascidos vivos tiveram a necessidade de UTI neonatal, e 0,9\% de óbitos.

Em estudo realizado por Neves (2011), fora evidenciado um apgar ótimo/excelente no quinto minuto em $93,9 \%$ recém-nascidos, $80,4 \%$ nasceram com um peso igual ou superior a $2.500 \mathrm{~g}$ e $3,6 \%$ dos recém-nascidos foram a óbito.

Tabela 02- Desfecho neonatal de recém-nascidos de puérperas em uma maternidade pública do estado do Amapá-AP. (PI)-2019. N:908

\begin{tabular}{|c|c|c|c|c|c|c|}
\hline & $\mathbf{N}$ & $\%$ & Média & Mín & Máx & Dp \\
\hline \multicolumn{3}{|c|}{ Classificação da escala do Apgar(Ao Nascer) } & 8,6 & 0,0 & 9,0 & 1,4 \\
\hline Ruim & 28 & $3,1 \%$ & & & & \\
\hline Regular & 44 & $4,8 \%$ & & & & \\
\hline Òtimo/Excelente & 837 & $92,1 \%$ & & & & \\
\hline \multicolumn{3}{|c|}{ Classificação da escala do Apgar(5 min) } & 10 & 0 & 10 & 1 \\
\hline Ruim & 10 & $1,1 \%$ & & & & \\
\hline Regular & 13 & $1,4 \%$ & & & & \\
\hline Òtimo/Excelente & 886 & $97,5 \%$ & & & & \\
\hline \multicolumn{7}{|l|}{ Peso neonatal } \\
\hline $500|-| 1.000 \mathrm{gr}$ & 4 & $0,40 \%$ & & & & \\
\hline $1001|-| 1.500 \mathrm{gr}$ & 17 & $1,90 \%$ & & & & \\
\hline $1501|-| 2000 \mathrm{gr}$ & 9 & $1,00 \%$ & & & & \\
\hline $2001|-| 2500 \mathrm{gr}$ & 37 & $4,10 \%$ & & & & \\
\hline$>2501 \mathrm{gr}$ & 842 & $92,60 \%$ & & & & \\
\hline \multicolumn{3}{|c|}{ Necessidade de uti neonatal } & & & & \\
\hline Sim & 43 & $4,70 \%$ & & & & \\
\hline Não & 866 & $95,30 \%$ & & & & \\
\hline \multicolumn{7}{|l|}{ Óbito neonatal } \\
\hline Sim & 8 & $0,90 \%$ & & & & \\
\hline Não & 901 & $99,10 \%$ & & & & \\
\hline
\end{tabular}

Fonte: Autor

$\mathrm{RC}: 34634$

Disponível em: https://www.nucleodoconhecimento.com.br/saude/desfecho-materno 
No que se refere a caracterização do desfecho neonatal em relação às características maternas (Tabela 3), nota-se que à localização da moradia que predominou o trabalho de parto prematuro foram os moradores que residem no interior do estado $15 \%$, seguido por $14,9 \%$ moradores da zona Norte da capital, $14,8 \%$ residem na zona Sul da capital. Não houve diferença significativa quanto a prematuridade a paciente ser do interior ou da capital. Esses dados são discordantes da maioria dos estudos, que atribui que uma maior taxa de prematuridade em pacientes residente no interior ${ }^{8}$. De acordo com estudos, estar associado à questão do acesso a aos serviços de saúde, considerando um estrutura de atendimento, localização e qualificação dos serviços, a maioria que reside em zona rural, ou seja, onde o acesso aos bens e serviços é restrito. Os fatores ambientais podem ser determinantes na taxa de mortalidade infantil ${ }^{8}$.

Em relação a faixa etária o trabalho de parto prematuro foi mais frequente em pacientes adultos $15,8 \%$. Os resultados indicam que a prematuridade prevaleceu nas pacientes que estudaram até ensino médio completo $23,4 \%$, com renda familiar maior que 3 salários mínimos 22,4\%. Dados controverso aos encontrados na literatura, consideram que, em geral, a adolescência, a baixa escolaridade e o baixo padrão socioeconômico podem predispor a situações potencialmente de risco para a mãe e o recém-nascido, além de impedir o acesso a informações e orientações, restringir a capacidade de cuidado e assistência e dificultar o exercício de direitos e cidadania ${ }^{9}$.

Com relação a história reprodutiva das pacientes o trabalho de parto prematuro se mostrou mais frequente em pacientes não multíparas $28,1 \%$. Um estudo realizado em Imperatriz-MA, a prematuridade foi mais frequente em primíparas ${ }^{1}$. No que se refere ao número de consultas e exames no pré-natal, o presente estudo demonstrou que a prematuridade foi mais frequente em paciente que não passaram por nenhuma consulta médica $16,3 \%$, que se consultaram somente uma vez com o enfermeiro $22,2 \%$ e naquelas que não realizaram os exames preconizados pelo MS $19,4 \%$. Observa-se que $46,3 \%$ das pacientes que não realizaram consulta médica ou realizaram no máximo 2 consultas tiveram partos prematuros, enquanto pacientes que realizaram 3 ou mais consultas 13,7\% tiveram parto prematuro. Em um estudo 
realizado no Paraná mostra que apenas 3\% das pacientes não realizaram consultas médicas ${ }^{9}$.

Tabela 03- Caracterização do desfecho neonatal em relação às características maternas segundo a cobertura da assistência ao pré-natal, início precoce e número de consultas realizadas em uma capital da região Norte do Brasil. (PI)-2019. N:908

\begin{tabular}{|c|c|c|c|c|}
\hline & \multicolumn{4}{|c|}{ Desfecho neonatal } \\
\hline & \multicolumn{2}{|c|}{ Parto a Termo } & \multicolumn{2}{|c|}{ Parto Prematuro } \\
\hline & $\mathrm{N}$ & $\mathrm{N}$ & $\mathrm{N}$ & $\%$ \\
\hline \multicolumn{5}{|l|}{ Região da residência: } \\
\hline Zona Norte & 275 & $85,1 \%$ & 48 & $14,9 \%$ \\
\hline Interior do Estado & 193 & $85,0 \%$ & 34 & $15,0 \%$ \\
\hline Zona Sul & 305 & $85,2 \%$ & 53 & $14,8 \%$ \\
\hline \multicolumn{5}{|l|}{ Idade (anos } \\
\hline$\leq 19$ anos (Adolescentes) & 164 & $88,6 \%$ & 21 & $11,4 \%$ \\
\hline$\geq 20$ anos (Adulto) & 609 & $84,2 \%$ & 114 & $15,8 \%$ \\
\hline \multicolumn{5}{|l|}{ Escolaridade Materna } \\
\hline E. Fundamental (Incompleto) & 256 & $88,0 \%$ & 35 & $12,0 \%$ \\
\hline E. Fundamental (Completo) & 55 & $90,2 \%$ & 6 & $9,8 \%$ \\
\hline E. Médio (Incompleto) & 140 & $84,8 \%$ & 25 & $15,2 \%$ \\
\hline E. Médio (Completo) & 242 & $83,7 \%$ & 47 & $16,3 \%$ \\
\hline E.Superior (Incompleto) & 31 & $81,6 \%$ & 7 & $18,4 \%$ \\
\hline E.Superior (Completo) & 49 & $76,6 \%$ & 15 & $23,4 \%$ \\
\hline \multicolumn{5}{|l|}{ Renda familiar } \\
\hline$<1 \mathrm{SM}$ & 394 & $86,8 \%$ & 60 & $13,2 \%$ \\
\hline $1|-2| \mathrm{SM}$ & 341 & $84,2 \%$ & 64 & $15,8 \%$ \\
\hline$\geq 3 S M$ & 38 & $77,6 \%$ & 11 & $22,4 \%$ \\
\hline \multicolumn{5}{|l|}{ № de gestações anteriores: } \\
\hline Nenhuma & 218 & $82,3 \%$ & 47 & $17,7 \%$ \\
\hline 1º Gestação & 188 & $86,2 \%$ & 30 & $13,8 \%$ \\
\hline
\end{tabular}




\begin{tabular}{|l|l|l|l|l|}
\hline $2^{\circ}$ Gestação & 144 & $85,7 \%$ & 24 & $14,3 \%$ \\
\hline$\geq 3^{\circ}$ Gestações & 222 & $86,7 \%$ & 34 & $13,3 \%$ \\
\hline Fez todos os exames preconizados pelo MS? & \\
\hline Sim & 640 & $86,1 \%$ & 103 & $13,9 \%$ \\
\hline Não & 133 & $80,6 \%$ & 32 & $19,4 \%$ \\
\hline Número de consultas médicas & & & \\
\hline Nenhuma & 180 & $83,7 \%$ & 35 & $16,3 \%$ \\
\hline 1 Consulta & 175 & $85,8 \%$ & 29 & $14,2 \%$ \\
\hline 2 Consultas & 160 & $84,2 \%$ & 30 & $15,8 \%$ \\
\hline$\geq 3^{\circ}$ Consultas & 258 & $86,3 \%$ & 41 & $13,7 \%$ \\
\hline Número de consultas com enfermeiro & & \\
\hline Nenhuma & 25 & $83,3 \%$ & 5 & $16,7 \%$ \\
\hline 1 Consulta & 147 & $77,8 \%$ & 42 & $22,2 \%$ \\
\hline 2 Consultas & 186 & $84,2 \%$ & 35 & $15,8 \%$ \\
\hline$\geq 3^{\circ}$ Consultas & 415 & $88,7 \%$ & 53 & $11,3 \%$ \\
\hline
\end{tabular}

Fonte: Autor

No que se refere ao Apgar no primeiro minuto (Tabela 4), constatou-se que recémnascidos com apgar ruim, 8,4\% das puérperas residem na zona norte da capital, eram adultas $8,2 \%$, que $14,7 \%$ estudaram até o ensino e 15,4\% tinham renda familiar de até 2 salários mínimos. Assim como recém-nascidos de pacientes secundigestas $8,7 \%$, que no momento do parto a idade gestacional inferior a 37 semanas de gestação, cujo não realizaram todos os exames preconizados pelo Ministério da saúde $10,3 \%$, não passaram por nenhuma consulta médica $9,3 \%$, que realizaram apenas uma consulta com o enfermeiro 10,6\% obtiveram um índice de Apgar ruim.

Em nosso estudo que somente a prematuridade e o parto cesariano foram estatisticamente significativo para um apgar ruim.

O índice de Apgar, largamente utilizado para mensurar a vitalidade do recém-nascido, avalia cinco sintomas objetivos: frequência cardíaca (ausente: 0; < 100/min: 1; > 100/min: 2); respiração (ausente: 0; fraca/irregular: 1; forte/choro: 2); tônus muscular 
(flácido: 0 ; flexão pernas: e braçõs: 1; movimento ativo/boa flexão: 2) e cor (cianótico/pálido: 0; cianose de extremidade: 1; rosado: 2). Importante indicador de risco para a morbimortalidade perinatal e neonatal a soma do Apgar varia 0 a $10^{7}$.

Tabela 04- Análise inferencial das características maternas segundo a cobertura da assistência ao pré-natal e a classificação da escala de APGAR, realizadas em uma capital da região Norte do Brasil. (PI)-2019. N:908

\begin{tabular}{|c|c|c|c|c|c|}
\hline & \multicolumn{4}{|c|}{$\begin{array}{l}\text { Classificação da escala do Apgar(Ao } \\
\text { Nascer) }\end{array}$} & \multirow[b]{3}{*}{$\begin{array}{l}\text { P- } \\
\text { valor }\end{array}$} \\
\hline & \multicolumn{2}{|c|}{ Ruim } & \multicolumn{2}{|c|}{ Ótimo/Excelente } & \\
\hline & $\mathrm{N}$ & $\%$ & $\mathrm{~N}$ & $\%$ & \\
\hline Região da residência: & & & & & 0,929 \\
\hline Zona Norte & 27 & $8,4 \%$ & 296 & $91,6 \%$ & \\
\hline Interior do Estado & 17 & $7,5 \%$ & 210 & $92,5 \%$ & \\
\hline Zona Sul & 28 & $7,8 \%$ & 330 & $92,2 \%$ & \\
\hline \multicolumn{2}{|l|}{ Idade (anos) } & & & & 0,611 \\
\hline$\leq 19$ anos (Adolescente) & 13 & $7,0 \%$ & 172 & $93,0 \%$ & \\
\hline$\geq 20$ anos (Adulto) & 59 & $8,2 \%$ & 664 & $91,8 \%$ & \\
\hline \multicolumn{2}{|l|}{ Escolaridade Materna } & & & & 0,658 \\
\hline $\begin{array}{l}\text { E. Fundamental } \\
\text { (Incompleto) }\end{array}$ & 19 & $6,5 \%$ & 272 & $93,5 \%$ & \\
\hline $\begin{array}{ll}\text { E. } & \text { Fundamental } \\
\text { (Completo) } & \end{array}$ & 5 & $8,2 \%$ & 56 & $91,8 \%$ & \\
\hline E. Médio (Incompleto) & 16 & $9,7 \%$ & 149 & $90,3 \%$ & \\
\hline E. Médio (Completo) & 21 & $7,3 \%$ & 268 & $92,7 \%$ & \\
\hline E.Superior (Incompleto) & 5 & $13,2 \%$ & 33 & $86,8 \%$ & \\
\hline E.Superior (Completo) & 6 & $9,4 \%$ & 58 & $90,6 \%$ & \\
\hline \multicolumn{2}{|l|}{ Renda familiar } & & & & 0,132 \\
\hline$<1 \mathrm{SM}$ & 43 & $9,5 \%$ & 411 & $90,5 \%$ & \\
\hline $1|-2| \mathrm{SM}$ & 24 & $5,9 \%$ & 381 & $94,1 \%$ & \\
\hline
\end{tabular}




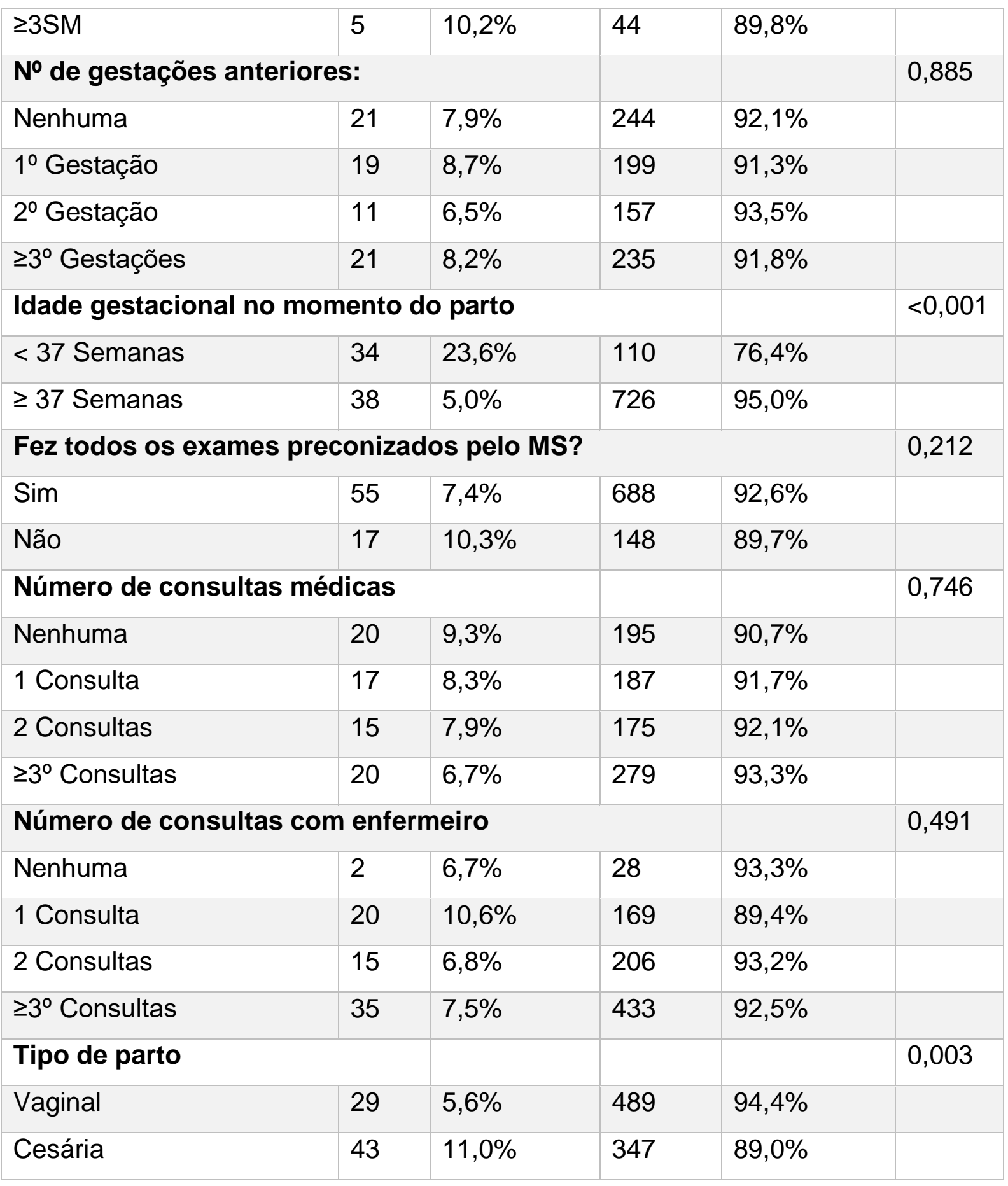

P-valor, Teste de associação Qui-quadrado, ao nível de significância de 5\%. Fonte: Autor.

Observou-se no estudo que aqui se propõe (Tabela 5) um desfecho neonatal desfavorável no que se refere ao índice de Apgar ruim ao nascer em RN prematuro 
$24,4 \%$, com peso variando entre $500 \mathrm{~g}$ a $1.000 \mathrm{~g}$, e aqueles que necessitaram de uti neonatal.

Os resultados obtidos demonstram os $\mathrm{RN}$ com apgar ruim tiveram necessidade de atendimento de maior nível de complexidade e maiores chances de complicações, remetendo à disponibilidade de estrutura técnica, equipamentos e recursos humanos capacitados para atendimento de maior complexidade e reversão da situação de risco apresentada ao nascer.

A organização Mundial da Saúde define baixo peso ao nascer o recém-nascido com peso ao nascimento menor que $2.500 \mathrm{~g}$, o baixo peso ao nascer sempre foi um motivo de preocupação para os profissionais de saúde, por se associar à maior mortalidade neonatal e infantil ${ }^{9}$.

Tabela 05- Análise Inferencial das características maternas segundo desfecho neonatal e da classificação da escala do APGAR, realizadas em uma capital da região Norte do Brasil. (PI)-2019. N:908.

\begin{tabular}{|c|c|c|c|c|c|}
\hline & \multicolumn{4}{|c|}{$\begin{array}{l}\begin{array}{l}\text { Classificação do Teste de Apgar(Ao } \\
\text { Nascer) }\end{array} \\
\end{array}$} & \\
\hline & \multicolumn{2}{|c|}{ Ruim } & \multicolumn{2}{|c|}{ Òtimo/Excelente } & \\
\hline & $\mathrm{N}$ & $\%$ & $\mathrm{~N}$ & $\%$ & P-valor \\
\hline \multicolumn{2}{|c|}{ Desfecho neonatal } & & & & $<0,001^{*}$ \\
\hline Parto a Termo & 39 & $5,0 \%$ & 735 & $95,0 \%$ & \\
\hline $\begin{array}{l}\text { Parto } \\
\text { Prematuro }\end{array}$ & 33 & $24,4 \%$ & 102 & $75,6 \%$ & \\
\hline \multicolumn{2}{|l|}{ Peso neonatal } & & & & $<0,001^{\star *}$ \\
\hline $500|-| 1.000 \mathrm{gr}$ & 4 & $100,0 \%$ & 0 & $0,0 \%$ & \\
\hline $1001|-| 1.500 \mathrm{gr}$ & 9 & $52,9 \%$ & 8 & $47,1 \%$ & \\
\hline $1501|-| 2000 \mathrm{gr}$ & 6 & $66,7 \%$ & 3 & $33,3 \%$ & \\
\hline $2001|-| 2500 \mathrm{gr}$ & 2 & $5,4 \%$ & 35 & $94,6 \%$ & \\
\hline$>2501 \mathrm{gr}$ & 51 & $6,1 \%$ & 791 & $93,9 \%$ & \\
\hline
\end{tabular}




\begin{tabular}{|c|c|c|c|c|c|}
\hline \multicolumn{3}{|c|}{ Necessidade de uti neonatal } & \multirow[b]{2}{*}{18} & \multirow[b]{2}{*}{$41,9 \%$} & $<0,001^{*}$ \\
\hline Sim & 25 & $58,1 \%$ & & & \\
\hline Não & 47 & $5,4 \%$ & 819 & $94,6 \%$ & \\
\hline
\end{tabular}

*P'valor, Teste de associação Qui Quadrado, ao nível de significação de 5\%

${ }^{* *}$ P-valor, Teste de associação Exato de Fisher, ao nível de significância de 5\%.

Fonte: Autor.

\section{CONCLUSÃO}

Concluímos que o desfecho neonatal desfavorável (Apgar Ruim) em parturientes atendidas em uma maternidade pública do Amapá foi estatisticamente significativo nas seguintes situações: a prematuridade, parto cesariano, necessidade de UTI neonatal e baixo peso ao nascer. Fatores como procedência (interior ou capital), idade materna, renda, escolaridade e paridade não influenciaram de forma significativa no desfecho neonatal.

\section{REFERÊNCIA}

1. ALMEIDA, A. C. Fatores de risco Materno para prematuridade em uma maternidade pública de Imperatriz-MA. Revista Gaúcha enfermagem, Porto Alegre, 2012.

2. BITTAR, R. E. Indicadores de risco para o parto prematuro. Revista Brasileira de Ginecologia e obstetrícia , São Paulo- SP, 27 abr. 2009.

3. BRASIL. MINISTÉRIO DA SAÚDE. Portaria №569/2000 - Dispõe sobre o Programa de Humanização no Pré-natal e nascimento. Diário Oficial da União, Brasília (DF); 8 de junho de 2000, seção 1, p.4-6. Disponível em <http://www.spp.org.br/Portaria_569_GM.pdf>. Acesso em 01 jul. 2019.

4. CASTRO, M.E; MOURA, M.A.V; SILVA, L.M.S. Qualidade da assistência prénatal: uma perspectiva das puérperas egressas. Revista RENE. v. 11. Número especial. p.73-81. 2010 
5. COIMBRA, L. C. Fatores associados à inadequação do uso da assistência prénatal. Revista de Saúde Pública, São Paulo- SP, 1 ago. 2013.

6. GONZAGA, I. C. A. Atenção pré-natal e fatores de risco associado à prematuridade e baixo peso ao nascer em capital do nordeste brasileiro. Ciência e saúde coletiva, Teresina-PI, 25 ago. 2015.

7. NEVES, A. F. G. B. Perfil epidemiológico dos neonatos nascidos em uma maternidade-escola na Paraíba. Dissertação (Mestrado em enfermagem) Universidade Federal da Paraíba, João Pessoa-PB, 2011.

8. PEREIRA, S. S. M. Perfil de gestantes acometidas de parto prematuro em uma maternidade pública. Revista Online de pesquisa, Rio de Janeiro- RJ, jul. 2018.

9. RAMOS, H. A. C. Fatores de risco para prematuridade: pesquisa documental. Escola Anna Nery revista de enfermagem, [S. I.], 6 abr. 2009.

10. VIELLAS, E. F. Assistência pré-natal no Brasil. Caderno saúde pública, Rio de Janeiro- RJ, 13 dez. 2013.

Enviado: Julho, 2019.

Aprovado: Julho, 2019. 